\title{
Cosmology with 3-D Gravitational Lensing
}

\author{
Andy Taylor \\ Institute for Astronomy, School of Physics, University of Edinburgh, \\ Royal Observatory, Blackford Hill, Edinburgh, EH9 3HJ, U.K. \\ email: ant@roe.ac.uk
}

\begin{abstract}
Weak gravitational lensing can be combined with distance information from galaxy redshifts to great effect in 3-D weak lensing. With both shear and redshift information I show how the full 3-D dark matter distribution can be reconstructed and present a first application to the COMBO-17 data-set. In addition, I describe the Jain-Taylor geometric test, taking ratios of galaxy shear at different distances, as a probe of the dark energy in the universe and present a preliminary application to COMBO-17.
\end{abstract}

\section{Introduction}

Gravitational lensing is usually thought of as a 2-D phenomenon, where the distances to galaxy images are averaged over and compressed into a radial weighting function. However, lensing is really a $3-\mathrm{D}$ effect, dependent on the relative geometry of the observer, lens and background source. By compressing the radial dimension, much information is lost. In the case of dark matter mapping, projection effects increase the uncertainty on images, while in statistical weak lensing information about the evolution of structure and gravitational lens geometry is mostly lost. This information can be particularly important, especially in the case of probing dark energy which directly affects both the evolution of structure and global geometry of the universe.

Depth information, from galaxy redshifts, has already been used in weak lensing studies to determine the median redshifts of the lens and background populations (see Brown et al 2003, who use the COMBO-17 data to determine the detailed population redshift distribution for a statistical shear analysis). But much more of this lost information can be regained by extending gravitational lensing to include the information about the radial positions of each of the background source galaxies.

Source distances will yield information on the distance to the lens. This is obvious when one considers a single, point-like lens. In this case the background shear pattern rises rapidly just behind the lens, flattening off at higher redshifts. By looking for the rise in the shear signal, by taking differences between shears at different redshifts, one can hope to pin down the redshift of the lens. What is perhaps less obvious is that there is an exact, one-to-one inversion between the shear pattern with distances to each source, and the full, 3-D distribution of the dark matter doing the lensing (Taylor, 2001). In Section 2, I outline the method used to achieve this inversion and present a first application to map the 3-D dark matter field in the COMBO-17 data-set.

In addition to mapping the 3 - $\mathrm{D}$ dark matter field, distance information is also valuable for more statistical tests. With distances one can extract the growth rate of dark matter evolution (see David Bacon in these proceedings) or add information in cosmological parameters (see Alan Heavens and Lindsay King's contributions). Here I describe an alternative method, the Jain-Taylor approach, based on extracting only geometric information and using it to probe the dark energy in the universe, free from assumptions about the dark matter. I begin with 3-D dark matter mapping. 


\section{3-D Dark Matter Mapping}

The central lensing equation relating the $3-\mathrm{D}$ Newtonian potential, $\Phi$, field to a gravitational lensing potential, $\phi$, is

$$
\phi(r, r \boldsymbol{\theta})=2 \int_{0}^{r} d r^{\prime}\left(\frac{r-r^{\prime}}{r r^{\prime}}\right) \Phi\left(r^{\prime}, r^{\prime} \boldsymbol{\theta}\right),
$$

where $\boldsymbol{\theta}$ is a position angle on the sky, and $r$ is the comoving distance. Here we have assumed a spatially flat universe, which can be easily relaxed, and the Born approximation. This just says that the lensing effect is due to deflections of the photon beam, characterized by the lensing potential, caused by the gravitational field of structure along the beam path. The lensing potential is related to the shear matrix, $\gamma_{i j}$, by

$$
\phi(r, r \boldsymbol{\theta})=2 \partial^{-4} \partial_{i} \partial_{j} \gamma_{i j}(r, r \boldsymbol{\theta}),
$$

where $\partial_{i} \equiv r\left(\delta_{i j}-\hat{r}_{i} \hat{r}_{j}\right) \nabla_{j}$ is a dimensionless differential operator on the sky and $\partial^{2} \equiv \partial_{i} \partial^{i}$ is its Laplacian. Equation (2.2) reconstructs the lensing potential up to a quadratic function on the sky with arbitrary function of distance (Taylor 2001, Bacon \& Taylor 2003). This arbitrary behaviour is due to the more familiar sheet-mass degeneracy for the lens convergence, translated to the lensing potential at each distance. This function can be estimated and removed from the data (see Bacon \& Taylor 2003).

It turns out that equation (2.1) can be exactly solved for the 3-D Newtonian potential,

$$
\Phi(r, r \boldsymbol{\theta})=\frac{1}{2} \partial_{r} r^{2} \partial_{r} \phi(r, r \boldsymbol{\theta}),
$$

where $\partial_{r}=\hat{\boldsymbol{r}} . \nabla$ is a radial derivative. This relation reflects the more intuitive idea that the lensing signal can be differenced to yield distance information about the lens. For completeness we note that a similar expression exists for the matter density field, $\delta$, and the convergence field, $\kappa$, given by

$$
\delta(r, r \boldsymbol{\theta})=\frac{2 a(r)}{3 \Omega_{m} H_{0}^{2}} r^{-2} \partial_{r} r^{2} \partial_{r} \kappa(r, r \boldsymbol{\theta}),
$$

where $a$ is the expansion factor.

The shot-noise on a reconstructed 3-D Newtonian potential field, per pixel, is given by

$$
\Delta \Phi=10^{-7}(n / 20 \text { sq.arcmin })^{-1 / 2}(\Delta z / 0.05)^{-5 / 2}(z / 0.1),
$$

where $n$ is the galaxy surface density, and is fairly independent of angular pixel size (Bacon \& Taylor, 2003). This is higher than the potential field we can expect for a small cluster, so we usually also apply a radial Wiener filter to help bring out the full structure (Bacon \& Taylor, 2003; Hu \& Keeton, 2002).

Not only is the $3-\mathrm{D}$ inversion of the lensing fields to the $3-\mathrm{D}$ matter fields straightforward, the quality of data needed to apply it is already available. The COMBO-17 data-set (Wolf et al, 2003) contains both lens quality galaxy images and accurate photometric redshift information $(\Delta z \approx 0.01)$. Putting the galaxy shear data on a $3 \mathrm{D}$ grid we can easily implement the 3-D inversion (see Taylor et al 2004 for details).

Figure 1 (top) shows the 3D distribution of the dark matter potential for the Abell 901/2 supercluster in the COMBO-17 survey. The main features of the supercluster are seen in the $x-y$ image (top-left), looking back towards the observer. Rotating the image we can see the main supercluster lies in a plane at redshift $z=0.17$, but a new cluster, $\mathrm{CB} 1$, lies behind the supercluster at $z=0.48$. The same structure is seen in the galaxy number density, displayed in similar orientations below. 

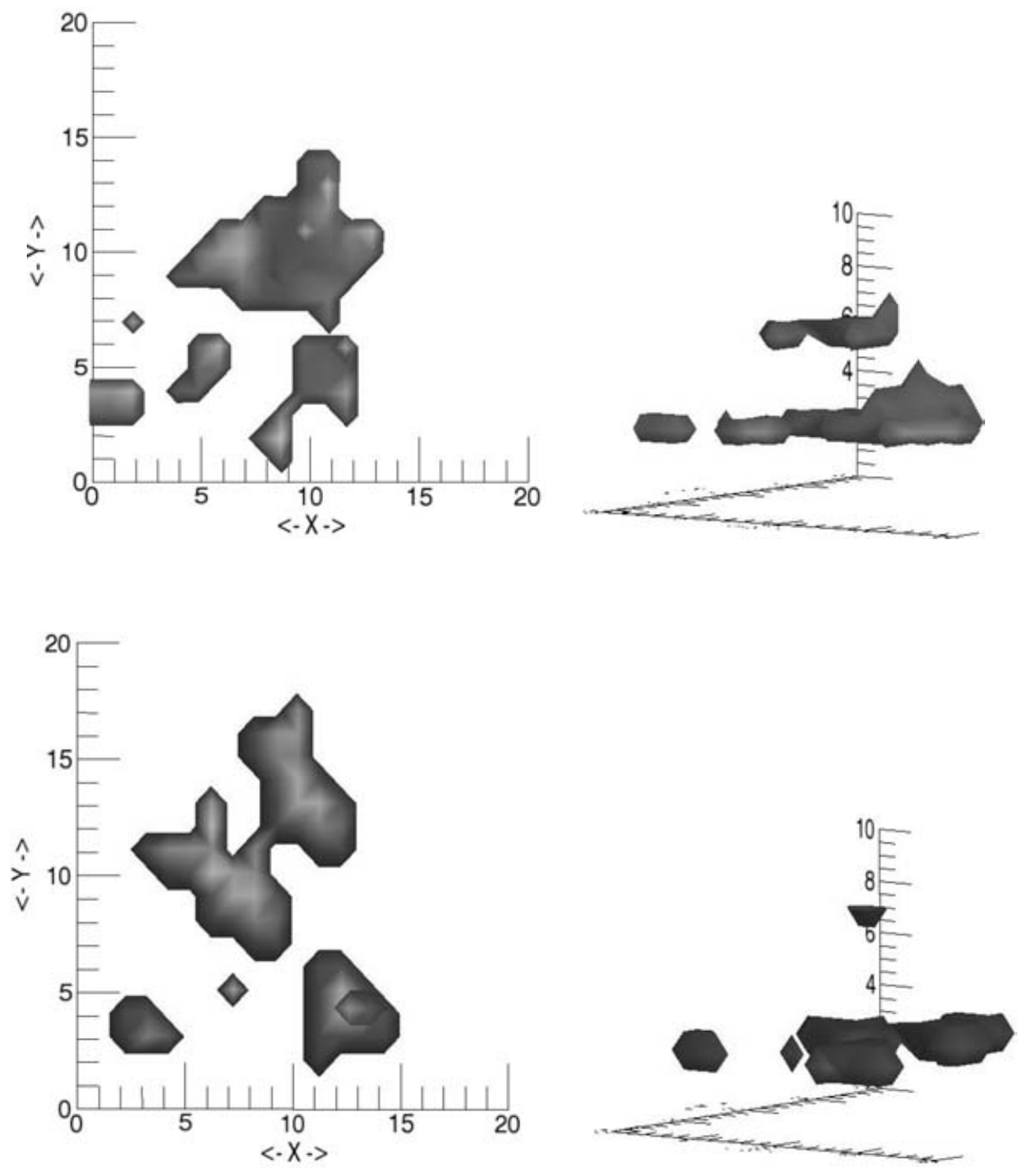

Figure 1. Three-dimensional iso-surface plots of the dark matter potential and galaxy number density fields from Taylor et al. (2004). The coordinates of the map are $(x, y, z)=\left(\theta_{x}, \theta_{y}, z\right)$, which distorts the map geometry. Note that axes are in pixel units, where $\Delta x=\Delta y=1.5$ arcmins and $\Delta z=0.05$ in redshift. The plots are filtered on the scale of the pixels. Upper panels: (LHS) The dark matter potential field, seen from high-redshift looking back to $z=0$ and (RHS) at an oblique angle. The supercluster A901/2 is seen as a sheet in the potential field in the lower part of the RHS map. The new cluster, CB1, is clearly seen as an isolated structure in the potential field behind A902 at $z=0.48$, with grid coordinates $(10,5,6)$. Lower panels: (LHS) The galaxy number density field for the A901/2 field, in the same projection as the above dark matter image, and (RHS) in oblique projection. The main overdensities due to the supercluster are seen as a sheet at low redshift, while the CB1 cluster is again clearly seen at $z=0.48$. 


\section{Dark Energy from the Geometric Test}

While the combination of shear and redshift information can be use to directly map the 3-D dark matter, applications can also be made to probe the 3-D statistical properties of weak shear. As well as a probe of dark matter, this can also be used to probe the dark energy component of the Universe. The dark energy makes itself felt via its effect on the evolution of the universe and in particular the Hubble parameter, $H(a)$. This affects both the evolution of dark matter, and the geometry of the Universe. See Alan Heavens and Lindsay King's contributions for more details. Bacon et al (2004 in preparation; see David Bacon in these proceedings) have fixed the cosmological model and used the 2-point shear correlation in 3-D to measure the evolution of the dark matter clustering.

An orthogonal statistic proposed by Jain and Taylor, the ratio of shears, can be used to measure purely the geometry of the universe, since the shear ratio only depends on the vacuum, matter and curvature density parameters $\Omega_{v}, \Omega_{m}, \Omega_{K}$, and the equation of state of the dark energy, $w=p / \rho$ (Jain \& Taylor, 2003). We can understand this by again thinking of an isolated, point-like lens. As the lens shear is proportional to the mass of the lens the ratio of shears at different redshifts is independent of the lens mass, and only depends on the ratio of comoving distances;

$$
R_{12}=\frac{\gamma_{1}(\theta)}{\gamma_{2}(\theta)}=\frac{\left(r_{1}-r_{\mathrm{L}}\right) / r_{1}}{\left(r_{2}-r_{\mathrm{L}}\right) / r_{2}}
$$

where $r_{\mathrm{L}}$ is the lens distance. The cosmological parameters enter via the comoving distance, $r(a)=\int_{0}^{a} d a^{\prime} / a^{\prime 2} H\left(a^{\prime}\right)$, where the Hubble parameter, $H(a)$, is given by

$$
H(a)=H_{0}\left[\Omega_{m} a^{-3}+\Omega_{K} a^{-2}+\Omega_{\mathrm{V}} e^{-3 \int_{1}^{a} d \ln a^{\prime}\left(1+w\left(a^{\prime}\right)\right)}\right]^{\frac{1}{2}},
$$

where $H_{0}$ is the Hubble parameter today.

Jain \& Taylor (2003) originally proposed this for ratios of shear correlations, but used the example of a single cluster as illustration. In fact the test can also be applied to individual clusters (since it is independent of structure), but we should also include the effect of other structure along the same path as an addition source of noise. Thus, a simple estimate for the fractional error on $w$ is;

$$
\frac{\Delta w}{w} \propto \frac{1}{M N_{\mathrm{bins}}} \sqrt{\frac{1}{N_{i} N_{\mathrm{cl}}}\left(\sigma_{\epsilon}^{2}+N_{i} C^{\gamma \gamma}\right)},
$$

where $M$ is the mass of the cluster, $N_{\text {bins }}$ is the number of background redshift bins with length of the redshift uncertainty, $N_{i}$ is the average number of galaxies per bin, $N_{\mathrm{cl}}$ is the number of clusters, $\sigma_{\epsilon}$ is the intrinsic galaxy ellipticity and $C^{\gamma \gamma}$ is the large-scale shear due to extra structure. Interestingly, this is only inversely proportional to the lens mass, suggesting that we should preferentially target massive clusters.

In Figure 2 we show the $\chi^{2}$ fit to $\Omega_{V}$ and $w$ from the ratios for the shear field in the background of the A901/2 supercluster in the COMBO-17 survey (Kitching, Taylor \& Bacon 2004, in preparation). We have assumed that the universe is spatially flat. No constraint is put on $\Omega_{V}$, as the data do not extend beyond $z=1$, but a (weak) constraint is found for $w$, from just three small clusters. For comparison we show the 1- and 2- $\sigma$ constraint on the $\Omega_{V}-w$ plane from WMAP $+2 \mathrm{dF}+$ Lyman- $\alpha$ data sets (Spergel et al, 2003). A single, large cluster could, in principle, provide a much stronger constraint on $w$. 


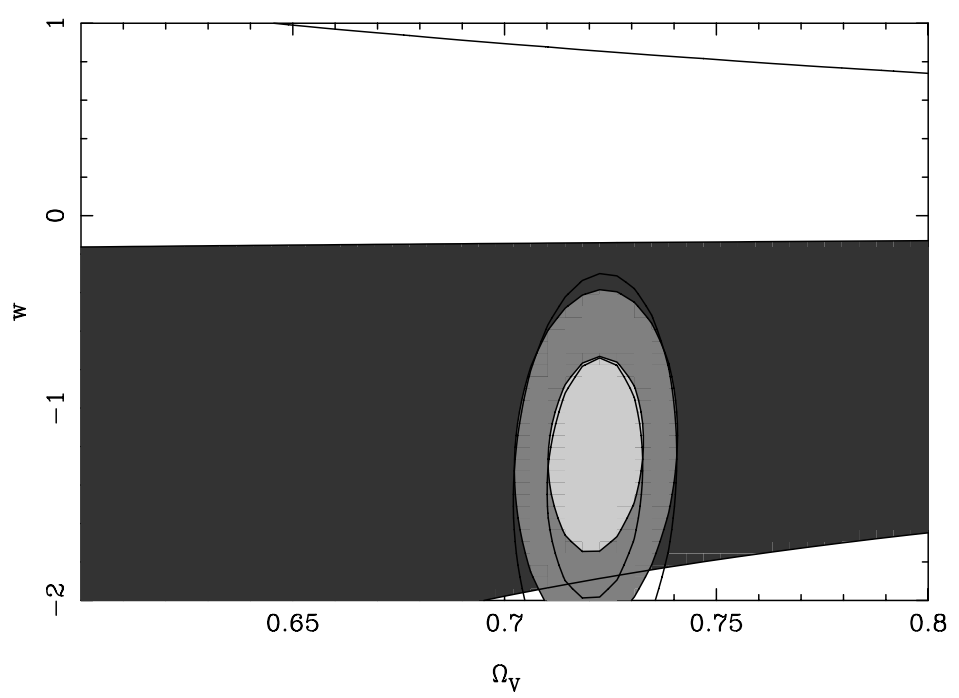

Figure 2. 1- $\sigma$ region in the $\Omega_{V}-w$ plane for the A901/2 supercluster field in the COMBO-17 survey (grey block). For comparison we also show the 1- and 2- $\sigma$ region from $W M A P+2 d F$, etc (solid ellipses) and the combined constraint (lighter grey-scale).

\section{Summary}

Combing weak shear with galaxy distance information, in $3-\mathrm{D}$ weak lensing, allows us to reconstruct the full $3-\mathrm{D}$ mass distribution of the universe. The exact inversion of the lens equation allows for a complete reconstruction, although the lensing kernel requires a double differentiation which amplifies the noise. This can be controlled by Wiener filtering. The data to apply this already exists, and I have shown the results for the A901/2 supercluster field from the COMBO-17 survey. In addition, 3-D weak lensing can be used for a purely geometric test by taking the ratios of galaxy shears behind galaxy clusters. This is only sensitive to the global geometry of the universe, and in particular the dark energy density and equation of state. Clearly, from these applications and many more, the availability of source redshifts and 3-D methods has opened up a whole new dimension for gravitational lensing.

\section{Acknowledgements}

Many thanks go to the Edinburgh Weak Lensers past and present, David Bacon, Meghan Gray, Michael Brown, Tom Kitching and Alan Heavens, the COMBO-17 team, especially Chris Wolf, Klaus Meisenheimer and Simon Dye, and to Bhuvnesh Jain for a very enjoyable collaboration. I also thank the PPARC for an Advanced Research Fellowship which supported this work.

\section{References}

Bacon D.J., Taylor A.N., 2003, MNRAS, 344, 1307

Brown M.L., Taylor A.N., Bacon D., Gray M., Dye S., Meisenheimer K. \& Wolf C. 2003, MNRAS, 341,100

Hu W., Keeton C. R., 2002, Phys. Rev. D, 66, 3506

Jain B., Taylor A.N., 2003, Phys. Rev. Lett., 91, 1302

Spergel D.N. et al., 2003, ApJS, 148, 175

Taylor A. N., 2001 (astro-ph/0111605) 
Taylor A. N., Bacon D.J., Gray M.E., Wolf C., Meisenheimer K., Dye S., Borch A., Kleinheinrich M., Kovacs M., Wisotzki L. 2004, MNRAS, in press (astro-ph/0402095)

Wolf C, Meisenheimer K., Rix H.-W., Borch A., Dye S., Kleinheinrich M. 2003, A\& $A$, 401, 73

\section{Discussion}

SARAH BRIDLE: Why are the uncertainties on the cluster redshifts from a parametric estimate of the cluster positions so large (see Taylor et al 2004), when the 3-D reconstruction of the mass field looks so good?

ANDY TAYLOR: There are a number of reasons for this. One is that a parametric fit tries to use the data non-locally, all along the line of sight. This means that an estimate of lens distance can get biased by poorer data and outliers at higher redshift. The 3-D reconstruction is more local. A second reason may also be that the Wiener filter is adding in information to the reconstruction, and this will help the reconstruction look better.

LAURA PARKer: At the end of your analysis will you be able to say anything about $w(z)$ (i.e. $\left.w^{\prime}\right)$ ?

ANDY TAYLOR: Unfortunately no. The geometric test is nearly as sensitive to $w^{\prime}$ as it is to $w$. But including $w^{\prime}$ introduces a strong correlation with $\Omega_{V}$, as both have a similar effect on the shear ratio, $R$. The data we have at the moment only weakly constrains $w$, so can't constraint another, more degenerate parameter.

LAURA PARKER: How big a data set would you require to start putting reasonable constraints on $w^{\prime}$ ?

ANDY TAYLOR: Jain \& Taylor (2003) showed that with a SNAP-type survey one could get $w^{\prime}$ to about $10 \%$. I suspect that the error on $w$ will fall rapidly in the next few years, so we might even start to see interesting constraints on $w^{\prime}$ with upcoming surveys in the not too distant future. 